\title{
Expression and significance of Annexin A3 in the osteosarcoma cell lines HOS and U2OS
}

\author{
XINXIN ZENG ${ }^{1 *}$, SHENGTAO WANG $^{2 *}$, PENG GUI $^{1}, \mathrm{HAO} \mathrm{WU}^{1}$ and ZHAOXU $\mathrm{LI}^{3}$ \\ Departments of ${ }^{1}$ Orthopaedics, and ${ }^{2}$ Emergency and Trauma Surgery, \\ Affiliated Hospital of Guilin Medical University, Guilin, Guangxi 541001; ${ }^{3}$ Department of Orthopaedics, \\ Nanxishan Hospital of Guangxi Zhuang Autonomous Region, Guilin, Guangxi 541002, P.R. China
}

Received October 26, 2018; Accepted April 17, 2019

DOI: $10.3892 / \mathrm{mmr} .2019 .10513$

\begin{abstract}
Annexin A3 (ANXA3) is highly expressed in different types of cancers, but the impact of ANXA3 in bone tumors is still not clear. In the present study, the expression of ANXA3 in osteosarcoma cells was first confirmed by cellular immunofluorescence. Reverse transcription-quantitative polymerase chain reaction (RT-qPCR) and western blot analysis were used to detect the expression of ANXA3 in osteoblasts in the osteosarcoma cell lines U2OS and HOS. Furthermore, small interfering (si)-RNA were transfected into U2OS and HOS cells via a liposome-mediated method. Then once ANXA3 had been successfully downregulated in U2OS and HOS cells, the cells were collected and total protein was extracted after $48 \mathrm{~h}$ of transfection. Western blot analysis was used to confirm successful ANXA3 transfection into osteosarcoma cells and the apoptotic rate of HOS and U2OS was detected by flow cytometry. The expression of ANXA3 in the osteosarcoma cell lines HOS and U2OS were first observed by confocal laser scanning microscopy, and was then detected by RT-qPCR and western blotting. The mRNA and protein levels of ANXA3 in the osteosarcoma cell lines HOS and U2OS were significantly increased compared with osteoblasts, particularly in HOS cells. When siRNA was transfected into HOS and U2OS cells, the protein expression level of ANXA3 was measured via western blotting. The results indicated that the expression of ANXA3 was significantly decreased. In addition, to determine whether ANXA3 knockdown induced cell apoptosis, the present study analyzed the apoptotic rate by flow cytometry. The results revealed that ANXA3 knockdown markedly increased HOS and U2OS cell apoptosis. To the best
\end{abstract}

Correspondence to: Dr Zhaoxu Li, Department of Orthopaedics, Nanxishan Hospital of Guangxi Zhuang Autonomous Region, 46 Chongxin Road, Guilin, Guangxi 541002, P.R. China

E-mail: 2240411900@qq.com

\section{*Contributed equally}

Key words: osteosarcoma, Annexin A3, apoptosis, molecular targeted therapy of our knowledge, the present study is the first to confirm that ANXA3 is highly expressed in the osteosarcoma cell lines HOS and U2OS. In addition, downregulation of ANXA3 expression in HOS and U2OS cells could increase apoptotic ability.

\section{Introduction}

Osteosarcoma (OS) is the most common type of malignant bone tumor in teenagers; a few patients are diagnosed at terminal stage, which is usually accompanied by lung metastases (1-3). The 5-year survival rate is $<70 \%$ with a high degree of malignancy and early metastasis (4). Despite accurate diagnoses, the preoperative administration of chemotherapy, operative treatments or even adjuvant chemotherapy after operation, 30-50\% of patients still develop distant metastasis. Fewer than $20 \%$ of patients survive 5 years after disease onset. To improve early diagnosis efficiency, novel therapeutic targets for implementing precision treatment are needed and individualized treatments for OS hold great promise. Therefore, investigation into the mechanisms of OS cell occurrence, invasion and metastasis may lead to the identification of potential molecular targets for OS treatment.

Annexin A3 (ANXA3) is a member of the Annexin superfamily (5), which plays a significant role in tumor occurrence, cell migration, immune regulation and drug-fasting (6). In mammalian cells, ANXA3 is usually localized to the cytoplasm, maintaining a stable form that can affect the cytoskeleton or proteins that mediate the cell-extracellular matrix, and are involved in cell transduction, differentiation, migration and apoptosis (7-8). In recent years, it has been shown that the overexpression of ANXA3 is associated with ovarian, lung, liver, prostatic, colorectal and pancreatic carcinoma (9-16), and with drug resistance (16-18). The abnormal expression of ANXA3 provides more direct methods for diagnosis, determining pathological stage and in the treatment of tumors, thus it has become a key area of research for treating tumors (19). However, the function and effect of ANXA3 on the regulation of OS cells has not been investigated. Therefore, determining the expression and significance of ANXA3 in OS cells might provide potential therapeutic targets for treating OS. To the best of our knowledge, the present study is the first to confirm that the overexpression of ANXA3 is closely associated with 
OS cell proliferation and apoptosis, as determined through a series of different experiments.

The present study investigated the role of ANXA3 in osteoblasts and the OS cell lines HOS and U2OS. First, it was confirmed that ANXA3 was expressed in osteoblasts as well as in HOS and U2OS cells. Secondly, ANXA3 was revealed to be significantly upregulated in HOS and U2OS cells. Finally, it was demonstrated that the inhibition of ANXA3 expression prevented tumor growth and promoted OS cell apoptosis. These results suggest a significant role for ANXA3 in the OS cell lines HOS and U2OS and highlight its potential application in OS therapy.

\section{Materials and methods}

Materials. Formaldehyde and Triton X-100 were purchased from Beijing Solarbio Science \& Technology Co., Ltd. and diluted to the indicated final concentrations. Anti-ANXA3 $(1: 1,000$; cat. no. ab33068) and mouse monoclonal anti- $\beta$-actin antibodies $(1: 2,000$; cat. no. ab8226) were obtained from Abcam. Horseradish peroxidase (HRP)-conjugated anti-rabbit secondary antibodies (1:5,000; cat. no. ZB2301) and HRP-conjugated anti-mouse secondary antibodies (1:5,000; cat. no. ZB2305) were obtained from ZSGB-BIO; OriGene Technologies, Inc. ProLong ${ }^{\circledR}$ Diamond Antifade Mountant and 4', 6-diamidino-2-phenylindole (DAPI) were obtained from Thermo Fisher Scientific, Inc. and the Lipofectamine $2000^{\mathrm{TM}}$ transfection reagent was obtained from Invitrogen; Thermo Fisher Scientific, Inc. The RNAprep pure Cell kit and the Transcriptor First Strand cDNA synthesis kit were purchased from Tiangen Biotech Co., Ltd. The RNA expression profiles of ANXA3 were obtained from The Cancer Genome Atlas (TCGA) database (www.cancer.gov/about-nci/organization/ $\mathrm{ccg} / \mathrm{research} /$ structural-genomics/tcga). Annexin Vphycoerythrin (PE)/7-aminoactinomycin D (AAD) Apoptosis Detection kit was obtained from BD Biosciences.

Cell culture. The human osteoblast cell line hFOB, and the human osteosarcoma cell lines HOS and U2OS were obtained from the Cell Collection of Chinese Academy of Sciences. HOS and U2OS cells were cultured in high-glucose Dulbecco's modified Eagle's medium (DMEM; Gibco; Thermo Fisher Scientific, Inc.) at $37^{\circ} \mathrm{C}$ with $5 \% \mathrm{CO}_{2}$. While hFOB cells were cultured in DMEM: Nutrient Mixture F-12 (DMEM/F12; Gibco; Thermo Fisher Scientific, Inc.) with $0.3 \mathrm{mg} / \mathrm{ml} \mathrm{G} 418$ (Beijing Solarbio Science \& Technology Co., Ltd.) at $33.5^{\circ} \mathrm{C}$ with $5 \% \mathrm{CO}_{2}$. All media were supplemented with $10 \%$ fetal bovine serum (Gemini Bio Products) and $100 \mu \mathrm{g} / \mathrm{ml}$ Penicillin-Streptomycin Solution (Beijing Solarbio Science \& Technology Co., Ltd.).

Immunofluorescence assay. Cells were seeded in 6-well plates at a density of $1 \times 10^{6}$ cells $/ \mathrm{ml}$ and fixed in formaldehyde $(100 \%)$ at $37^{\circ} \mathrm{C}$ for $5 \mathrm{~min}$, after which they were allowed to attach for $2 \mathrm{~h}$. Next, $0.1 \%$ Triton $\mathrm{X}-100$ was added to the plates for $5 \mathrm{~min}$ and blocked for $1 \mathrm{~h}$ at $37^{\circ} \mathrm{C}$ with $1 \% \mathrm{BSA}$ (Thermo Fisher Scientific, Inc.) They were then washed with $0.01 \mathrm{~mol} / 1$ phosphate buffer solution (PBS) and incubated with anti-ANXA3 (1:1,000; cat. no. ab33068; Abcam) overnight at $4^{\circ} \mathrm{C}$ on a shaking table. Incubation with Goat Anti-Mouse
Immunoglobulin G (cat. no. ab6708; Abcam) was performed for $1 \mathrm{~h}$ at room temperature, and then cells were stained with DAPI dihydrochloride for $3 \mathrm{~min}$ at room temperature. Then Antifade Mounting Medium (Thermo Fisher Scientific, Inc.) was added to cells at $4^{\circ} \mathrm{C}$ for $5 \mathrm{~min}$ in the dark following washing with $\mathrm{ddH}_{2} \mathrm{O}$ and the staining results were observed by laser confocal microscopy (Carl Zeiss AG; magnification, $\mathrm{x} 40)$.

Reverse transcription-quantitative polymerase chain reaction $(R T-q P C R)$ analysis. Osteoblasts were planted in a culture bottle with DMEM/F12 medium while HOS and U2OS cells were cultured with DMEM. Total RNA was extracted with the RNAsimple total RNA kit (Tiangen Biotech Co., Ltd.) from cells then reverse transcribed to cDNA using a FastQuant RT kit (Tiangen Biotech Co., Ltd.) at $42^{\circ} \mathrm{C}$ for $15 \mathrm{~min}$, followed by two incubation at $95^{\circ} \mathrm{C}$ for $3 \mathrm{~min}$ and at $4^{\circ} \mathrm{C}$ for $5 \mathrm{~min}$. Assessment of gene expression was performed using a SYBR Green PCR kit (Takara Bio, Inc.) using the iCycler (Bio-Rad Laboratories, Inc.) according to the manufacturer's instructions. Briefly, RNase Free $\mathrm{ddH}_{2} \mathrm{O}$ was added into $2 \mu \mathrm{l}$ of $5 \mathrm{X}$ gDNA Eraser Buffer, $1 \mu \mathrm{l}$ of gDNA Eraser, $1 \mu \mathrm{l}$ of Total RNA up to $10 \mu \mathrm{l}$, and vortex mixed at $42^{\circ} \mathrm{C}$ for $15 \mathrm{~min}$, followed by storage at $-20^{\circ} \mathrm{C}$. Next, $1 \mu 110 \mathrm{X}$ FastRT Buffer, $2 \mu 1 \mathrm{RT}$ Primer Mix and $2 \mu \mathrm{l} \mathrm{FQ-RT} \mathrm{Primer} \mathrm{Mix} \mathrm{were} \mathrm{added} \mathrm{as} \mathrm{well}$ as RNase Free $\mathrm{ddH}_{2} \mathrm{O}$ to a total volume of $10 \mu \mathrm{l}$, followed by gentle mixing. PCR was performed by activating the DNA polymerase at $95^{\circ} \mathrm{C}$ for $10 \mathrm{~min}$, followed by 40 cycles of $95^{\circ} \mathrm{C}$ for $15 \mathrm{sec}$ and $60^{\circ} \mathrm{C}$ for $45 \mathrm{sec}$, and a final extension at $75^{\circ} \mathrm{C}$ for $10 \mathrm{~min}$. The gene primer sequences were as follows: For human ANXA3, forward 5'-CCCATCAGTGGATGCTG AAG-3' and reverse 5'-TCACTAGGGCCACCATGAGA-3'; and GAPDH (the internal control), forward 5'-GGATATTGT TGCCATCAATGACCT-3' and reverse 5'-AGCCTTCTC CATGGTGGTGAAGA-3'. Finally, gene expression data were evaluated according to the $2^{-\Delta \Delta \mathrm{Cq}}$ method (20).

Western blot analysis. Proteins were extracted using RIPA lysis buffer (Beyotime Institute of Biotechnology), then cells were centrifuged at $12,000 \mathrm{x}$ g for $20 \mathrm{~min}$ at $4^{\circ} \mathrm{C}$. Supernatants containing 20-30 $\mu \mathrm{g}$ protein were collected, and the protein content was quantified using a BCA protein assay kit (Thermo Fisher Scientific, Inc.). Samples (20 $\mu \mathrm{g} / \mathrm{lane})$ were separated by $12 \%$ SDS-PAGE and transferred to PVDF membranes. The blots were blocked with 5\% skim milk in TBST for $2 \mathrm{~h}$ at room temperature, incubated with primary anti-ANXA3 $(1: 1,000)$ or anti- $\beta$-actin $(1: 2,000)$ primary antibodies at $4^{\circ} \mathrm{C}$ overnight. After washing with TBST (0.05\% Tween-20), the membranes were incubated with HRP-conjugated anti-rabbit secondary antibodies $(1: 5,000)$ or HRP-conjugated anti-mouse secondary antibodies $(1: 5,000)$ at $27^{\circ} \mathrm{C}$ for $2 \mathrm{~h}$. The bands were visualized using an enhanced chemiluminescence detection kit (Tiangen Biotech Co., Ltd.) and quantified by Quantity One software (version 2.4; Bio-Rad Laboratories, Inc.).

Cell transfection. Small interfering RNA (siRNA) ANXA3-651 (forward 5'-GAA AUC UUA ACU ACC AGGATT-3', reverse 3'-UCCUGGUAGUUA AGAUUUCTT-5'), siRNA ANXA3-732 (forward 5'-GAUGACAUUAGUUCCGAA ATT-3', reverse 3'-UUUCGGAACUAAUGUCAUCTT-5'), 
siRNA ANXA3-1094 (forward 5'-GAAGGGUAUUGGAAC UGAUTT-3', reverse 3'-AUCAGUUCCAAUACCCUUCTT-5') and negative control (NC) siRNA (5'-UUCUCCGAACGUGUC ACGUTT-3') were designed and synthesized by the Whitehead Institute for Biomedical Research. GAPDH (forward 5'-UGA CCUCAACUACAUGGUUTT-3', reverse 3'-TTACUGGAG UUGAUGUACCAA-5') was used as positive control, negative control siRNA (forward 5'-UUCUCCGAACGUGUCACG UTT-3', reverse 3'-TTAAGAGGCUUGCACAGUGCA-5') was used as negative control, and a no-transfection group was used as the blank control. In order to inhibit the expression of ANXA3, cells at 50-60\% confluence were seeded overnight and transfected with $50 \mathrm{nM}$ siRNAs targeting ANXA3 or control siRNAs using Lipofectamine 2000 (Invitrogen; Thermo Fisher Scientific, Inc.) in OptiMEM (Invitrogen; Thermo Fisher Scientific, Inc.) according to the manufacturer's protocol. After $48 \mathrm{~h}$ of transfection, cells were collected for subsequent research.

Flow cytometric analysis. After $48 \mathrm{~h}$ of transfection, cells were plated in 6-well plates at a density of $1 \times 10^{5}$ for $24 \mathrm{~h}$, then harvested and washed with ice-cold PBS (Beijing Solarbio Science \& Technology Co., Ltd.). All cells were digested with EDTA-free typsin (Beijing Solarbio Science \& Technology Co., Ltd.) and stained with the Annexin V-PE/7-AAD apoptosis detection kit (BD Biosciences), and incubated in the dark for $15 \mathrm{~min}$ at room temperature. Apoptosis was analyzed using CellQuest software (version 5.1; BD Biosciences) with a FACSCalibur flow cytometer (BD Biosciences).

Statistical analysis. All quantitative assays were performed in triplicate and statistical analysis was performed using SPSS 18.0 software (IBM Corp.). Experimental data were expressed as the mean \pm standard deviation. Comparisons in datasets containing multiple groups were conducted using one-way analysis of variance followed by Tukey's post-hoc test. $\mathrm{P}<0.05$ was considered to indicate a statistically significant difference.

\section{Results}

ANXA3 expression in the OS cell lines HOS and U2OS. In order to determine whether ANXA3 was expressed in osteoblasts, and the OS cell lines HOS or U2OS, an immunofluorescence assay was performed and the results were observed by laser confocal microscopy. The results showed that ANXA3 was expressed in osteoblasts, and HOS and U2OS cells (Fig. 1), and was markedly expressed in both HOS and U2OS cells.

ANXA3 mRNA expression levels in osteoblast, and the OS cell lines HOS and U2OS. In order to investigate whether ANXA3 mRNA was expressed in osteoblasts, and HOS and U2OS cells, the present study performed RT-qPCR. The results revealed that ANXA3 mRNA was expressed in the OS cell lines HOS and U2OS as well as in osteoblasts (Fig. 2). The mRNA levels were significantly upregulated in both HOS and U2OS cells compared with the osteoblasts; ANXA3 expression in HOS cells was increased by $\sim 2.99$-fold and in U2OS cells levels were also increased by 1.25 -fold when compared with osteoblasts (Fig. 2). Therefore, the subsequent experiments were designed based on these results.
ANXA3 protein expression levels in osteoblasts, and the OS cell lines HOS and U2OS. In order to determine whether ANXA3 protein was involved in osteoblasts, and HOS and U2OS cells, the extracted proteins were examined and quantified by western blotting. The results demonstrated that ANXA3 was significantly increased in HOS and U2OS cells when compared with osteoblasts (Fig. 3A). Furthermore, the protein expression of ANXA3 in HOS cells increased by 3.25 -fold, and increased by $\sim 2.33$-fold in U2OS cells (Fig. 3B).

ANXA3 decreases apoptosis in the HOS and U2OS OS cell line. On the basis of the aforementioned results, it was concluded that the expression of ANXA3 in the OS cell lines HOS and U2OS was significantly different when compared with the osteoblast group. In addition, ANXA3 in HOS cells was significantly increased when compared with U2OS cells as determined by RT-qPCR and western blot analyses. To investigate whether the expression levels of ANXA3 were associated with the apoptotic rate, the present study downregulated ANXA3 via siRNA transfection for $48 \mathrm{~h}$ and the collected cells were examined via western blotting, RT-qPCR and flow cytometry.

The present results indicated that ANXA3 was significantly decreased in both HOS and U2OS cells (Fig. 4A). Compared with the blank control group, in HOS cells the protein expression of the siRNA651 group decreased to 0.26 -fold, whereas both the siRNA732 and siRNA1094 groups decreased to 0.31 -fold. In addition, the positive control group decreased to 0.37 -fold and the $\mathrm{NC}$ group exhibited a 0.87 -fold decrease $(\mathrm{P}<0.05$; Fig. 4B). In U2OS cells, compared with the blank control group, the protein expression of ANXA3 in the siRNA651 group decreased to 0.21-fold, the siRNA732 group decreased to 0.27 -fold and the siRNA1094 group decreased to 0.26 -fold. In addition, the positive control group decreased to 0.32 -fold, and the $\mathrm{NC}$ group caused a 0.81 -fold decrease ( $\mathrm{P}<0.05$; Fig. 4B).

The mRNA expression levels of ANXA3 in HOS cells, compared with the blank control group, in the siRNA651 group was decreased by $\sim 0.37$-fold, the siRNA732 group was approximately decreased to 0.43 -fold, and the siRNA1094 group was approximately decreased to 0.44 -fold. The positive control group was decreased to $\sim 0.47$-fold, and the NC group was decreased to $\sim 0.91$-fold $(\mathrm{P}<0.05$; Fig. $4 \mathrm{C})$. The mRNA expression levels of ANXA3 in the U2OS cells, when compared with the blank control group, in the siRNA651 group were decreased to $\sim 0.33$-fold, the siRNA732 group was decreased to $\sim 0.39$-fold, and the siRNA1094 group was decreased $\sim 0.44$-fold. The positive control group was decreased to $\sim 0.43$-fold, and the negative control group was decreased to $\sim 0.89$-fold ( $\mathrm{P}<0.05$; Fig. $4 \mathrm{C}$ ).

The apoptotic rate in the HOS cells of the control group was $8.1 \%$, whereas in the siRNA651 group it was $21.3 \%$, for the siRNA732 group it was $13.55 \%$, and in the siRNA1094 group it was $13.82 \%$, respectively. In addition, in U2OS cells, the apoptotic rate for the control group was 7.4\%, whereas in the siRNA651 group it was $21.08 \%$, in the siRNA732 group it was $13.63 \%$, and in the siRNA1094 group it was $12.52 \%$ $(\mathrm{P}<0.05 ;$ Fig. 5). In the osteoblast group, the apoptotic rate in the control group was $2.76 \%$, while in the siRNA651 group 

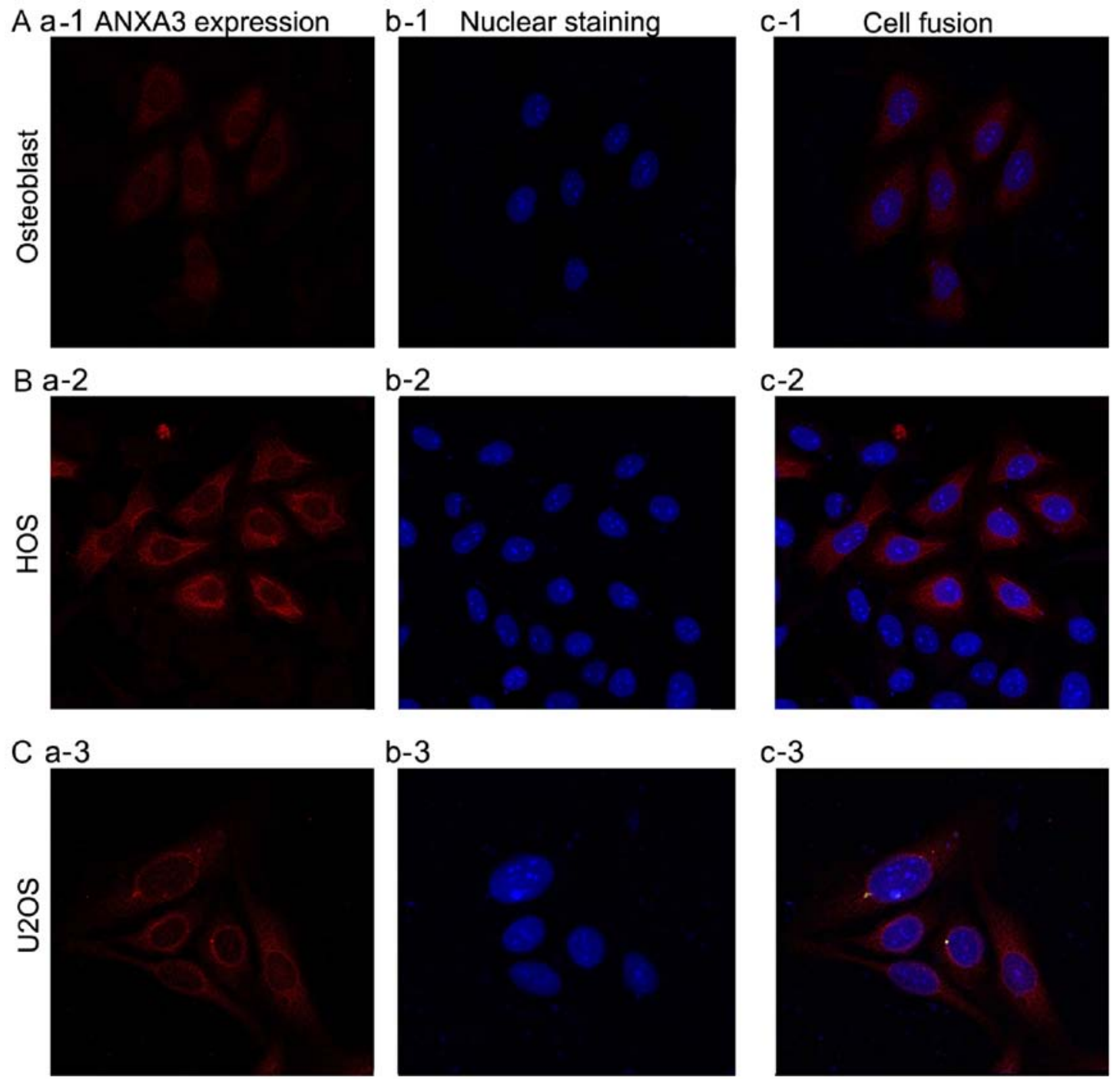

b-3
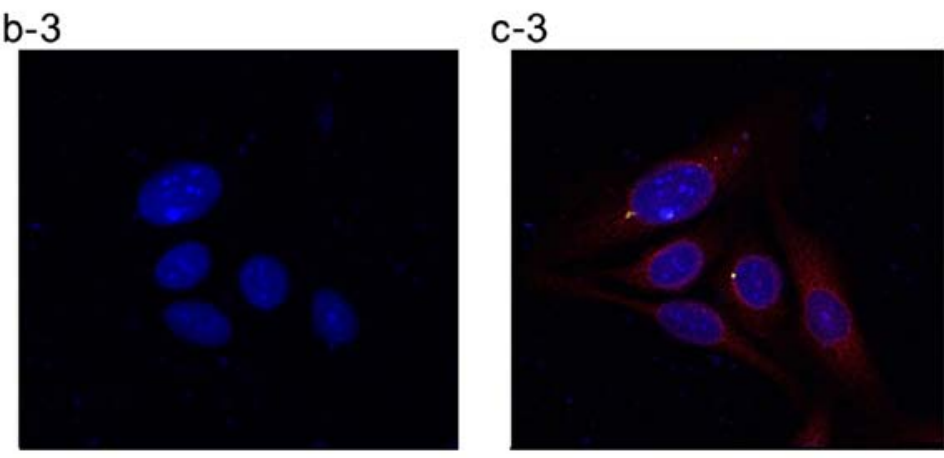

Figure 1. ANXA3 is expressed in osteoblasts as well as the OS cell lines HOS and U2OS. (A) Osteoblasts, (B) HOS cells and (C) U2OS cells were stained with anti-ANXA3 and then with secondary antibody. The results were examined and photographed by laser confocal microscopy. (a1-3) Red staining indicates ANXA3 protein expression and (b1-3) blue staining represents nuclear staining; (c1-3) the merged images are also presented. Magnification, x40. ANXA3, Annexin A3; OS, osteosarcoma.

it was $2.75 \%$, in the siRNA732 group it was $2.75 \%$ and in the siRNA1094 group it was $2.78 \%$ ( $P>0.05$; Fig. 5). The results suggested that ANXA3 downregulation may increase apoptotic rates of the OS cell lines HOS and U2OS but it had no significant effect on osteoblasts.

\section{Discussion}

The results of the present study demonstrated that ANXA3 was expressed in the OS cell lines HOS and U2OS as well as osteoblasts. Notably, the ANXA3 expression levels in HOS and U2OS were significantly increased when compared with osteoblasts. In addition, the present study further confirmed that downregulation of AXNA3 induced apoptosis. Thus, the present findings suggest that the expression of ANXA3 may be critically related to OS cells, and inhibiting ANXA3 may be an effective method for OS therapy.

A previous study reported that the abnormal expression of ANXA3 has been observed in various types of tumors and is associated with cancer progression making it a novel

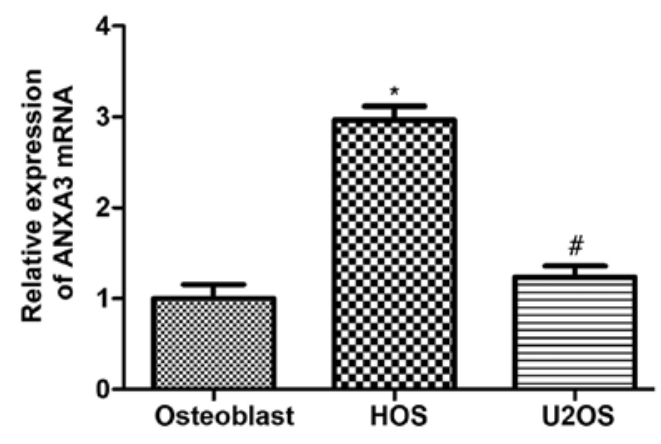

Figure 2. Gene expression of ANXA3 in osteoblasts, and the osteosarcoma cell lines HOS and U2OS. Cells $\left(10^{6}\right.$ per well) were cultured for $48 \mathrm{~h}$, and then total RNA was collected and examined by reverse transcription-quantitative polymerase chain reaction. Data are presented as the mean \pm standard deviation $(n=3) .{ }^{*} \mathrm{P}<0.05$ vs. osteoblast group; ${ }^{\text {"P}} \mathrm{P}<0.05$ vs. HOS group. ANXA3, Annexin A3.

therapeutic target (21). For example, several studies have reported that ANXA3 activated the JNK signaling pathway of 

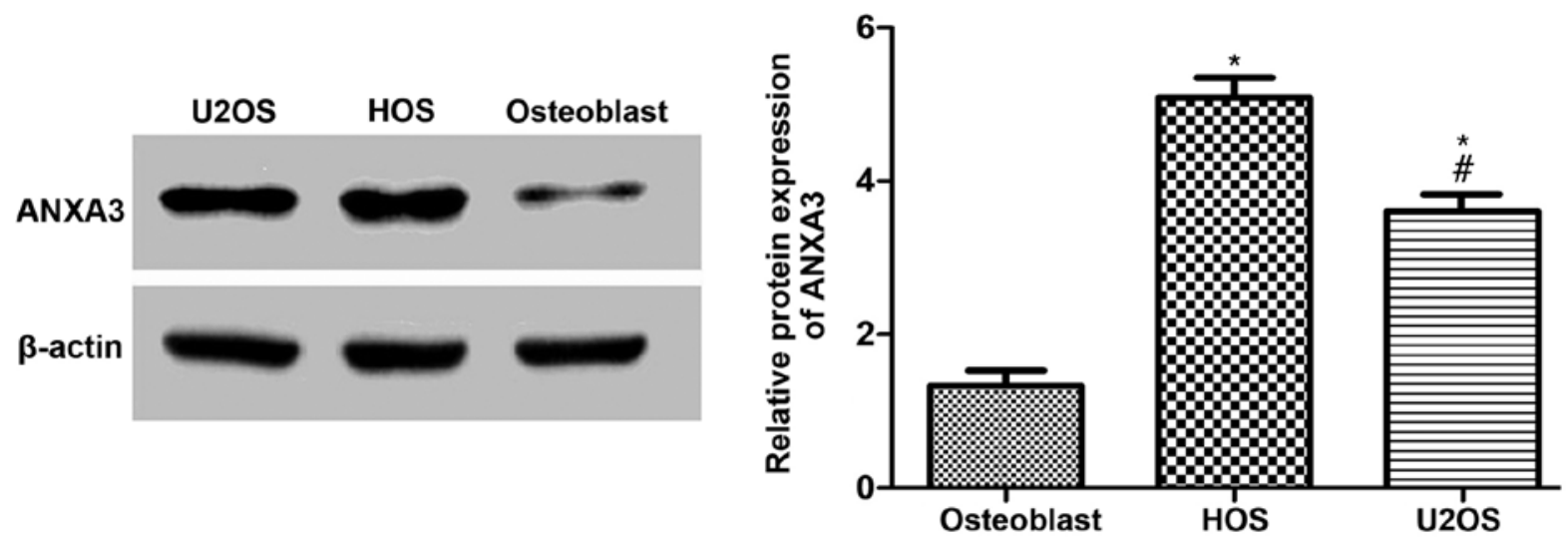

Figure 3. Protein expression levels of ANXA3 in the osteosarcoma cell lines, HOS and U2OS, and osteoblasts. Cells ( $10^{6}$ per well) were cultured for $48 \mathrm{~h}$, then total proteins were extracted and measured by western blotting. $\beta$-actin was used as the internal control. Data are presented as the mean \pm standard deviation $(\mathrm{n}=3)$. ${ }^{*} \mathrm{P}<0.05$ vs. osteoblast group; ${ }^{\# \mathrm{P}}<0.05$ vs. HOS group. ANXA3, Annexin A3.

A

HOS

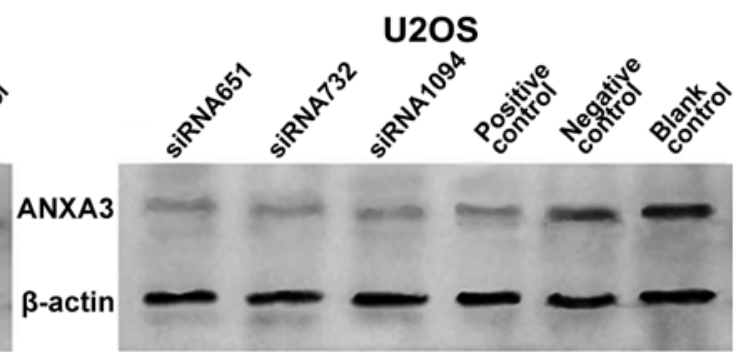

\section{B}
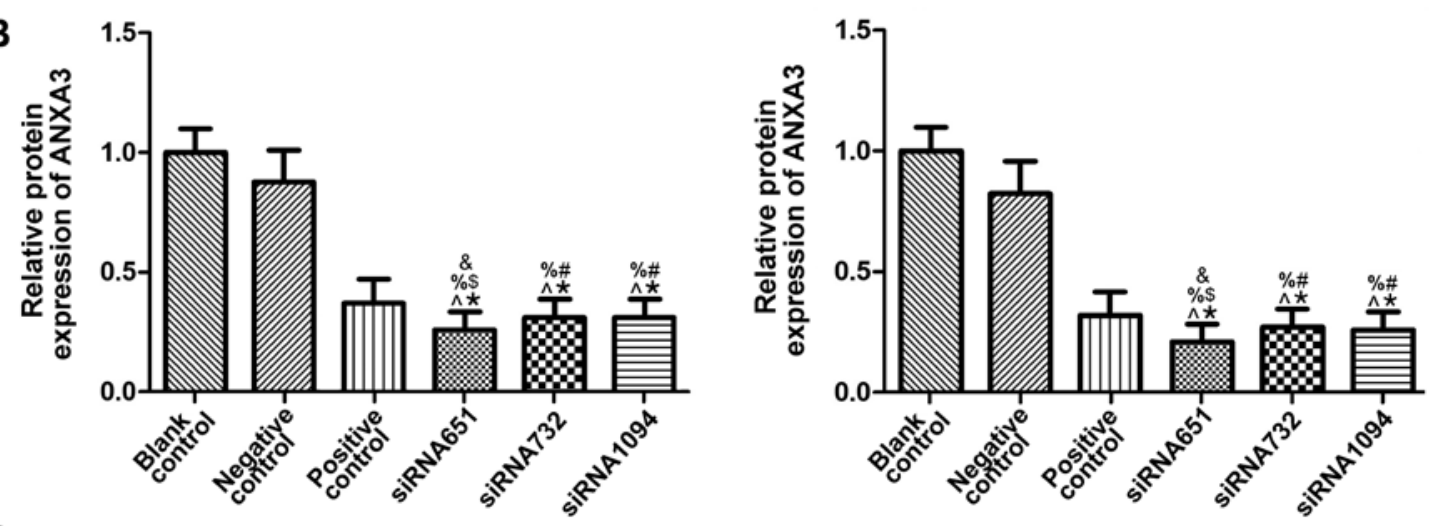

C
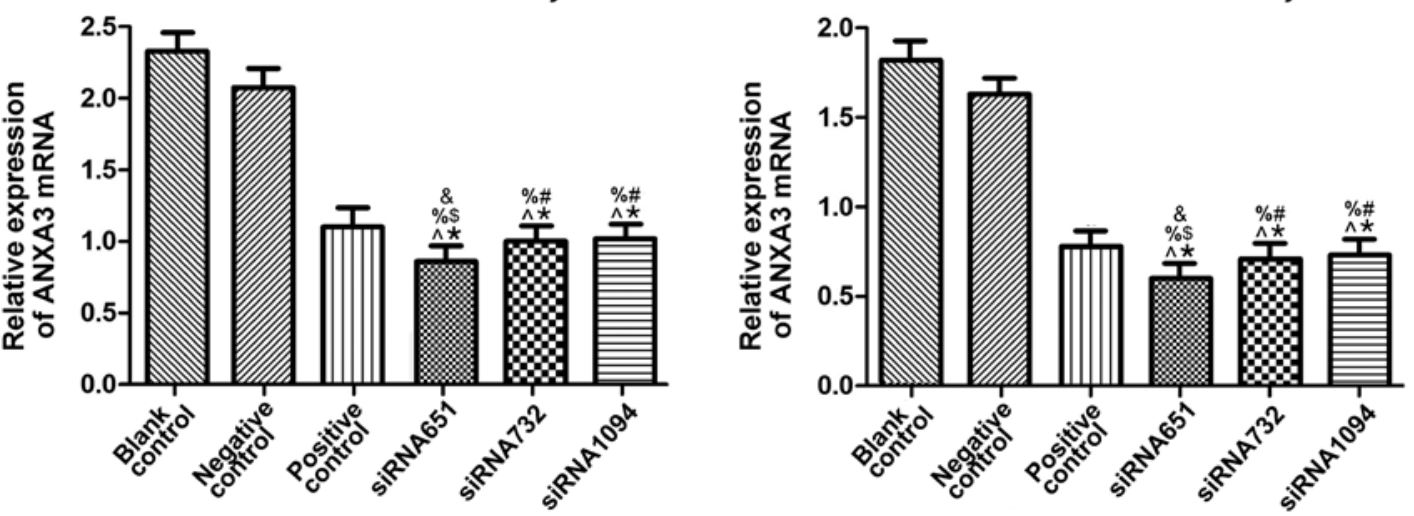

Figure 4. Impact of ANXA3 downregulation on its protein and mRNA expression in the osteosarcoma cell lines, HOS and U2OS. (A-C) Cells (10 ${ }^{6}$ per well) were cultured for $48 \mathrm{~h}$, then total proteins were extracted and measured by (A and B) western blotting, and total RNA was collected and examined by (C) reverse transcription-quantitative polymerase chain reaction. $\beta$-actin was used as the internal control. Data are presented as the mean \pm standard deviation ( $\mathrm{n}=3$ for each group). ${ }^{\mathrm{P}}<0.05$ vs. Negative Control group; ${ }^{\%} \mathrm{P}<0.05$ vs. Positive Control group; ${ }^{\circ} \mathrm{P}<0.05$ vs. Blank Control group; ${ }^{\text {}} \mathrm{P}<0.05$ vs. siRNA651 group; ${ }^{\$} \mathrm{P}<0.05$ vs. siRNA732 group; ${ }^{*} \mathrm{P}<0.05$ vs. siRNA1094 group. ANXA3, Annexin A3; siRNA, small interfering RNA.

stress-activated proteins in hepatocellular carcinoma (22-24). Furthermore, previous studies have demonstrated that the
JNK signaling pathway activated $\mathrm{CD} 133^{+}$glioblastoma stem cell self-renewal to promote hepatocellular carcinoma 

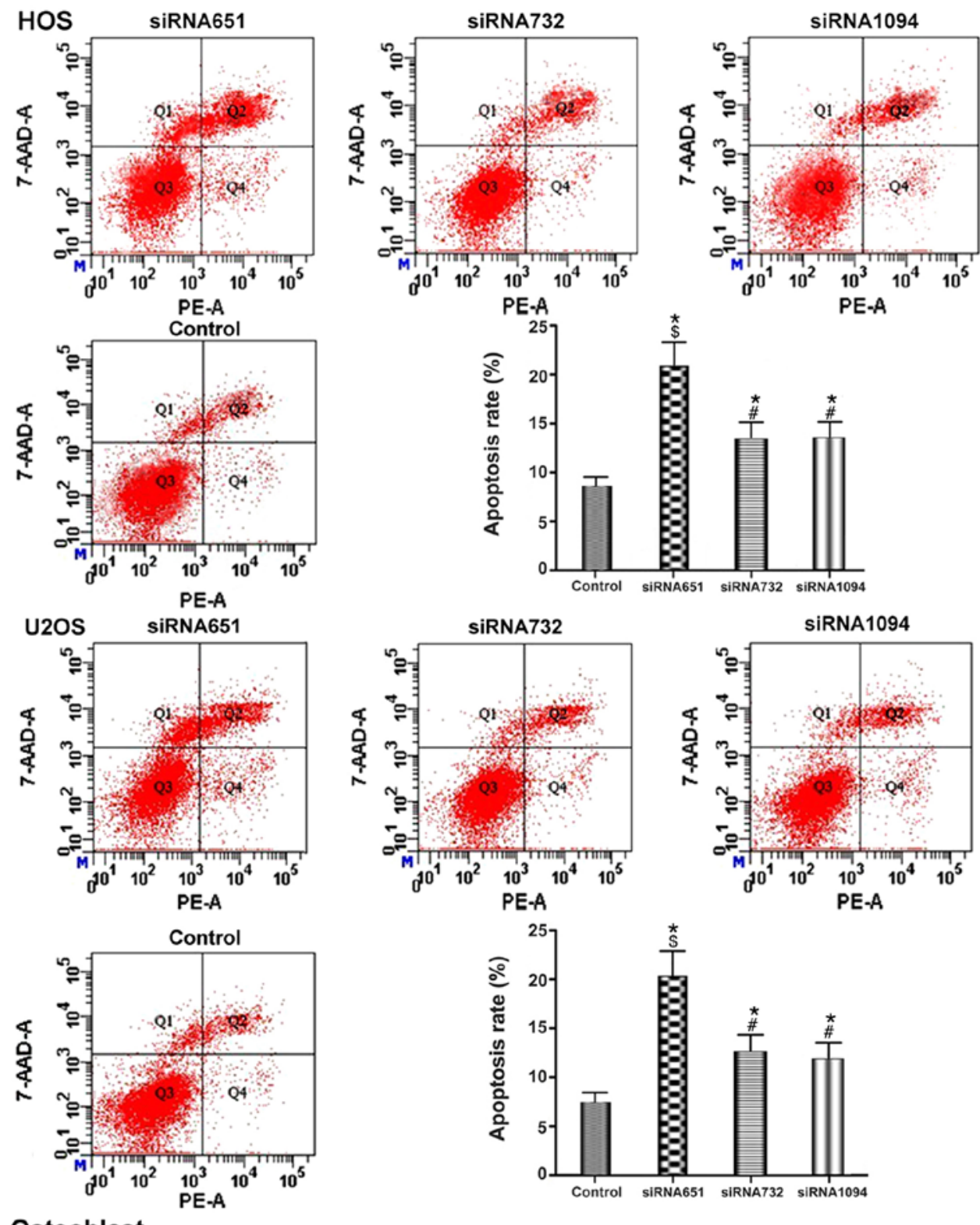

\section{Osteoblast siRNA651}
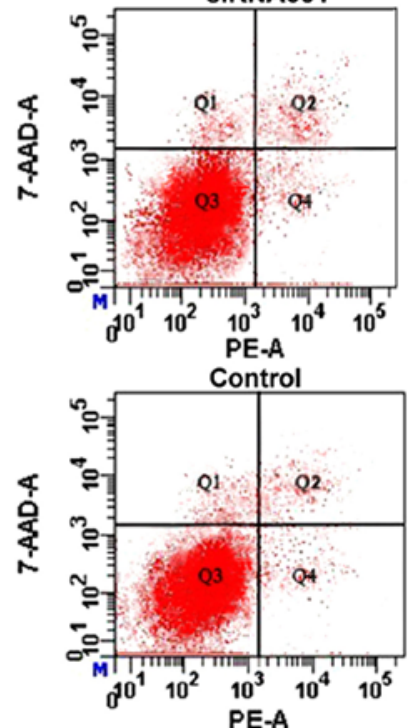
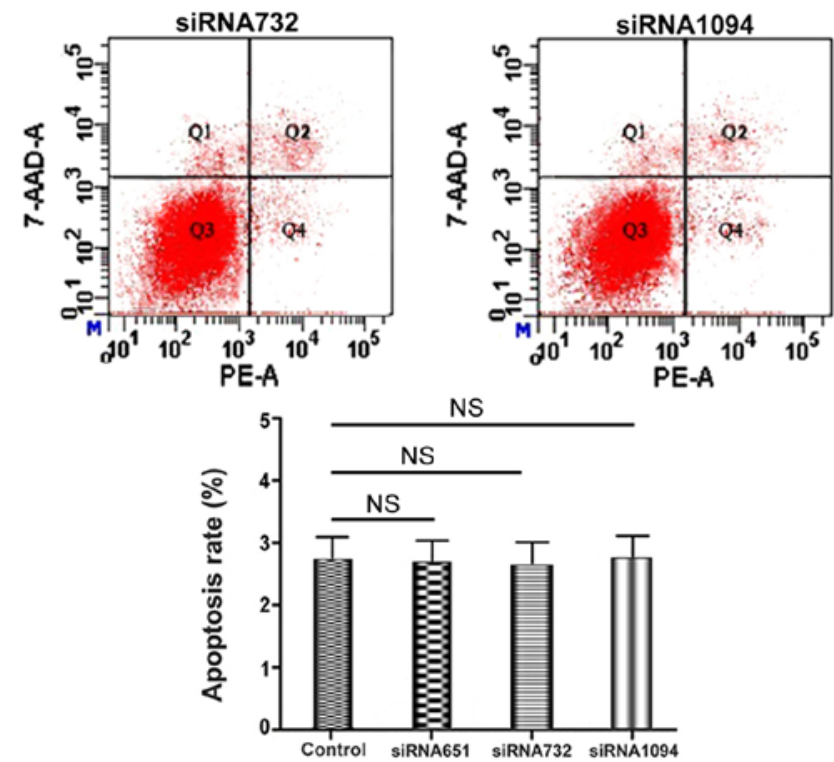

Figure 5. Impact of the downregulation of ANXA3 on apoptosis in the osteosarcoma cell lines HOS and U2OS, and osteoblasts. Apoptotic rates in the control, siRNA651, siRNA732 and siRNA1094 groups were detected via flow cytometry. Data are presented as the mean \pm standard deviation (n=3 for each group). ${ }^{*} \mathrm{P}<0.05$ vs. Control group; ${ }^{\prime} \mathrm{P}<0.05$ vs. siRNA651 group; ${ }^{\mathrm{P}} \mathrm{P}<0.05$ vs. siRNA732 group. NS, not significant; ANXA3, Annexin A3; siRNA, small interfering RNA; PE, phycoerythrin; 7-AAD, 7-aminoactinomycin D. 
differentiation (25-27). The JNK signaling pathway plays a significant role in ANXA3-mediated cancer stem cells. It has been reported that blocking JNK pathway reduced the expression of ANXA3 as determined by RT-qPCR; this result suggested that the expression levels of ANXA3 in hepatocellular carcinoma cells may be positively correlated with the JNK pathway (12). Further study confirmed that transfection of dendritic cells with ANXA3 activated autologous $\mathrm{CD}^{+} \mathrm{T}$ cells and $\mathrm{CD}^{+} \mathrm{T}$ cells to kill $\mathrm{CD}^{+} 33^{+}$hepatocellular carcinoma cells (28). Other previous studies have suggested that increased ANXA3 expression may be associated with the drug resistance of ovarian cancer $(16,29)$, poor prognosis of breast cancer $(29,30)$ and increased lymphatic metastasis (31).

Previous studies have also confirmed that ANXA3 is highly correlated with many types of tumors indicating that the activation of ANXA3 may increase proliferative activity in various types of cancers (32-35). A new therapeutic method that could inhibit ANXA3 could also decrease the activation of relevant types of cancers. The present data suggested that the expression of ANXA3 may be positively correlated with OS and demonstrated the previously mentioned hypothesis. Therefore, ANXA3 is expected to become a new target for OS therapy.

The traditional Chinese medicine celastrol is a natural pentacyclic triterpene, extracted from the Thunder God Vine and has an anti-cancer effect in many malignant tumors. However, its potential anti-cancer mechanism in OS requires further investigation. Our previous studies have shown that $\gamma \delta$ T cell immunotherapy has a positive effect on OS (4,36-39), and the results also indicated that celastrol increased OS cell lysis by $\gamma \delta \mathrm{T}$ cells via the upregulation of death receptor (40). This result indicated that celastrol may be able to induce primary OS cell death in vivo for $\gamma \delta \mathrm{T}$ cell lysis. It is hypothesized that ANXA3 downregulation via treatment with celastrol may increase the killing effect of $\gamma \delta$ T cells on OS cells. The mechanism of celastrol in ANXA3 will be further examined in the future.

Taken together, the present results indicated that ANXA3 was significantly overexpressed in the OS cell lines HOS and U2OS, and downregulated AXNA3 expression increased the apoptotic ability of HOS and U2OS cells. The present results suggested that anti-ANXA3 has a potential function as an inhibitor of OS. Therefore, ANXA3 could be used in the early diagnosis of and intervention of bone tumors as a novel target for OS therapy. However, further investigation into the underlying mechanisms is required.

\section{Acknowledgements}

Not applicable.

\section{Funding}

The present study was supported by National Natural Science Foundation of China (grant no. 81360400).

\section{Availability of data and materials}

The datasets used and/or analyzed during the current study are available from the corresponding author upon reasonable request.

\section{Authors' contributions}

ZL conceived and designed the study. XZ performed the experiments and was the primary contributor in writing the manuscript. SW conducted the data analyses. PG and HW collected the data and produced all of the figures. All authors read and approved the final manuscript.

\section{Ethics approval and consent to participate}

Not applicable.

\section{Patient consent for publication}

Not applicable.

\section{Competing interests}

The authors declare that they have no competing interests.

\section{References}

1. Michaelis J: Osteosarcoma. Lancet 1: 1174, 1988.

2. Kansara M, Teng MW, Smyth MJ and Thomas DM: Translation biology of osteosarcoma. Nat Rev Cancer 14: 722-735, 2014.

3. Gianferante DM, Mirabello L and Savage SA: Germline and somatic genetics of osteosarcoma-connecting aetiology, biology and therapy. Nat Rev Endocrinol 13: 480-491, 2017.

4. Li ZX, Wang RY and Tang JC: Sodium valproate enhance $\gamma \delta \mathrm{T}$ cells killing osteosarcoma. Chin J Exp Surg 33: 154-157, 2016.

5. Perron B, Lewit-Bentley A, Geny B and Russo-Marie F: Can enzymatic activity, or otherwise, be inferred from structural studies of annexin III? J Biol Chem 272: 11321-11326, 1997.

6. Gerke V, Creutz CE and Moss SE: Annexins: Linking Ca2+ signalling to membrane dynamics. Nat Rev Mol Cell Biol 6: 449-461, 2005.

7. Moss SE and Morgan RO: The annexins. Genome Biol 5: 219, 2004.

8. Gerke V and Moss SE: Annexins: From structure to function. Physiol Rev 82: 331-371, 2002.

9. Hamelin-Peyron C, Vlaeminck-Guillem V, Haidous H, Schwall GP, Poznanovic S, Gorius-Gallet E, Michles S, Larue A, Guillotte M, Ruffion A, et al: Prostate cancer biomarker annexin A3 detected in urines obtained following digital rectal examination presents antigenic variablility. Clin Biochem 47: 901-908, 2014.

10. Wu N, Liu S, Guo C, Hou Z and Sun MZ: The role of annexin A3 playing in cancers. Clin Transl Oncol 15: 106-110, 2013.

11. Zeidan B, Jackson TR, Larkin SE, Cutress RI, Coulton GR, Ashton-Key M, Murray N, Packham G, Gorgoulis V, Garbis SD and Townsend PA: Annexin A3 is a mammary marker and potential neoplastic breast cell therapeutic target. Oncotarget 6: 21421-21427, 2015

12. Tong M, Fung TM, Luk ST, Ng KY, Lee TK, Lin CH, Yam JW, Chan KW, Ng F, Zheng BJ, et al: ANXA3/JNK signaling promotes self-renewal and tumor growth, and its blockade provides a therapeutic target for hepatocellular carcinoma. Stem Cell Reports 5: 45-59, 2015.

13. Liu YF, Xiao ZQ, Li MX, Zhang PF, Li C, Li F, Chen YH, Yi H, Yao HX and Chen ZC: Quantitative promote analysis reveals annexin A3 as a novel biomarker in lung adenocarcinoma. J Pathol 217: 54-64, 2009.

14. Liu YF, Chen YH, Li MY, Zhang PF, Li GQ, Xiao ZQ and Chen ZC: Quantitative proteomic analysis identifying three annexins as lymph node metastasis-related proteins in lung adenocarcinoma. Med Oncol 29: 174-184, 2012.

15. Pan QZ, Pan K, Weng DS, Zhao JJ, Zhang XF, Wang DD, Lv L, Jiang SS, Zheng HX and Xia JC: Annexin A3 promotes tumorigenesis and resistance to chemotherapy in hepatocellular carcinoma. Mol Carcinog 54: 598-607, 2015.

16. Yan X, Yin J, Yao H, Mao N, Yang Y and Pan L: Increased expression of annexin A3 is a mechanism of platinum resistance in ovarian cancer. Cancer Res 70: 1616-1624, 2010. 
17. Tong SW, Yang YX, Hu HD, An X, Ye F, Hu P, Ren H, Li SL and Zhang DZ: Proteomic investigation of 5-flourouracil resistance in a human hepatocellular carcinoma cell line. J cell Biochem 113: 1671-1680, 2012.

18. Pénzváltó Z, Tegze B, Szász AM, Sztupinszki Z, Likó I, Szendrői A, Schäfer R and Győrffy B: Identifying resistance mechanisms against five tyrosine kinase inhibitors targeting the ERBB/RAS pathway in 45 cancer cell lines. PLoS One 8: e59503, 2013.

19. Li ZX: Potential of human $\gamma \delta \mathrm{T}$ cells for immunotherapy of osteosarcoma. Mol Biol Rep 40: 427-437, 2013.

20. Livak KJ and Schmittgen TD: Analysis of relative gene expression data using real-time quantitative PCR and the 2(-Delta Delta C(T)) method. Methods 25: 402-408, 2001.

21. Mussunoor S and Murray GI: The role of annexins in tumor development and progression. J Pathol 216: 131-140, 2008.

22. Shao P, Qu WK, Wang CY, Tian Y, Ye ML, Sun DG, Sui JD, Wang LM, Fan R and Gao ZM: MicroRNA-205-5p regulates the chemotherapeutic resistance of hepatocellular carcinoma cells by targeting PTEN/JNK/ANXA3 pathway. Am J Transl Res 9: 4300-4307, 2017.

23. Yang YM, Kim SY and Seki E: Inflammation and liver cancer: Molecular mechanisms and therapeutic targets. Semin Liver Dis 39: 26-42, 2019.

24. Li Z, Zhang L, Gao M, Han M, Liu K, Zhang Z, Gong Z, Xing L, Shi $\mathrm{X}, \mathrm{Lu} \mathrm{K}$ and $\mathrm{Gao} \mathrm{H}$ : Endoplasmic reticulum stress triggers xanthoangelol-induced protective autophagy via activation of JNK/c-Jun Axis in hepatocellular carcinoma. J Exp Clin Cancer Res 38: 8, 2019.

25. Kim JB, Lee S, Kim HR, Park SY, Lee M, Yoon JH and Kim YJ: Transforming growth factor- $\beta$ decreases side population cells in hepatocellular carcinoma in vitro. Oncol Lett 15: 8723-8728, 2018.

26. Jin Y, Mao J, Wang H, Hou Z, Ma W, Zhang J, Wang B, Huang Y, Zang S, Tang J and Li L: Enhanced tumorigenesis and lymphatic metastasis of CD133+ hepatocarcinoma ascites syngeneic cell lines mediated by JNK signaling pathway in vitro and in vivo. Biomed Pharmacother 67: 337-345, 2013.

27. Hagiwara S, Kudo M, Nagai T, Inoue T, Ueshima K, Nishida N, Watanabe T and Sakurai T: Activation of JNK and high expression level of CD133 predict a poor response to sorafenib in hepatocellular carcinoma. Br J Cancer 106: 1997-2003, 2012.

28. Pan Q, Pan K, Wang QJ, Weng DS, Zhao JJ, Zheng HX, Zhang XF, Jiang SS, Lv L, Tang Y, et al: Annexin A3 as a potential target for immunotherapy of liver cancer stem-like cells. Stem Cells 33: $354-366,2015$.
29. Zhou T, Li Y, Yang L, Yang L, Tang T, Zhang L and Shi J: Annexin A3 as a prognostic biomarker for breast cancer: A retrospective study. Biomed Res Int 2017: 2603685, 2017.

30. Zeng C, Ke Z, Song Y, Yao Y, Hu X, Zhang M, Li H and Yin J: Annexin A3 is associated with a poor prognosis in breast cancer and participates in the modulation of apoptosis in vitro by affecting the Bcl-2/Bax balance. Exp Mol Pathol 95: 23-31, 2013.

31. Liu YF, Xiao ZQ, Li MX, Li MY, Zhang PF, Li C, Li F, Chen YH, Yi H, Yao HX and Chen ZC: Quantitative proteome analysis reveals annexin A3 as a novel biomarker in lung adenocarcinoma. J Pathol 217: 54-64, 2009.

32. Li J, Zhou T, Liu L, Ju YC, Chen YT, Tan ZR and Wang J: The regulatory role of Annexin 3 in nude mouse bearing a subcutaneous xenograft of MDA-MB-231 human breast carcinoma. Pathol Res Pract 214: 1719-1725, 2018.

33. Jeun M, Park S, Kim Y, Choi J, Song SH, Jeong IG, Kim CS and Lee KH: Self-Normalized detection of ANXA3 from untreated urine of prostate cancer patients without digital rectal examination. Adv Healthc Mater: 6, 2017.

34. Liu YF, Liu QQ, Zhang YH and Qiu JH: Annexin A3 knockdown suppresses lung adenocarcinoma. Anal Cell Pathol (Amst) 2016: 4131403, 2016.

35. Zhou T, Li Y, Yang L, Liu L, Ju Y and Li C: Silencing of ANXA3 expression by RNA interference inhibits the proliferation and invasion of breast cancer cells. Oncol Rep 37: 388-398, 2017.

36. Li ZX, Wang RY and Tang JC: Type I IFN-mediated enhancement of anti-osteosarcoma cytotoxicity of human $\gamma \delta \mathrm{T}$ cells. Chin J Immun 11: 1533-1535, 1542, 2014

37. Li Z, Zhang J, Tang J and Wang R: Celastrol increases osteosarcoma cell lysis by $\gamma \delta \mathrm{T}$ cells through up-regulation of death receptors. Oncotarget 7: 84388-84397, 2016.

38. Li Z,Xu Q, Peng H,Cheng R, SunZ and YeZ: IFN- $\gamma$ enhances HOS and U2OS cell lines susceptibility to $\gamma \delta \mathrm{T}$ cell-mediated killing through the Fas/Fas ligand pathway. Int Immunopharmacol 11: 496-503, 2011.

39. Li Z, Peng H, Xu Q and Ye Z: Sensitization of human osteosarcoma cells to V $\gamma 9 \mathrm{~V} \delta 2$ T-cell-mediated cytotoxicity by zoledronate. J Orthop Res 30: 824-830, 2012.

40. Li ZX, Zhang JZ, Wang ST, Wang RY and Tang JC: Celastrol increases osteosarcoma cells line HOS lysis by $\gamma \delta$ T cells through TRAIL way. Chin J Immun 32: 1777-1780, 2016.

This work is licensed under a Creative Commons Attribution-NonCommercial-NoDerivatives 4.0 International (CC BY-NC-ND 4.0) License. 